\title{
INDIVIDUALISED COLLEGE TEACHING
}

\author{
James A. Kulik and Chen-Lin C. Kulik \\ University of Michigan, \\ Ann Arbor, Michigan 48109, USA
}

Individualised instruction has grown in importance in education in the last few decades. Its increasing importance is clearly shown by the rise in the number of publications in this area in recent years. The Education Index listed four or five articles per year on individualised instruction during the forties and fifties, 35 articles per year during the sixties, and over a hundred articles a year during the seventies (Kozak, 1974). The growth in interest in individualised instruction has been especially dramatic at the college level. After Skinner's (1954) development of teaching machines, college teachers began experimenting with various techniques for individualising college teaching, including programmed learning, computer-assisted instruction and total system of self-paced learning such as Keller's Personalised System of Instmetion or PSI (Keller, 1968) and Postlethwait's AudioTuLorial Approach or A-T (Postlethwait, Novak and Murray, 1972).

Conventional reviews on individualised college teaching seldom reach firm conclusions about its effectiveness. The reticence of reviewers about this matter is not due to a lack of evaluation of experiments in individualised instruction. Individualised teaching has been evaluated time and again. The problem rather is that each evaluation has been carried out in a specific setting and the different evaluations produced different results. The findings defy easy summary and conventional reviews have not managed to fit the pieces together to form a coherent picture.

In an effort to synthesise findings on individualised college teaching, we began applying Glass's (1976) meta-analytic techniques to the literature in 1977. So far we have carried out analyses of four major approaches to individualised teaching:

1. programmed instruction

2. computer-assisted instruction

3. Keller's l'ersonalised System of Inctmetion, and

4. Postlethwait's Aidio-Tutorial Aprroach.

A detailed presentation of our results appears in a series of reports (Kulik, Kulik and Cohen, 1979a, 1979b, 1979c; Kulik, Cohen and Ebeling, in press). This paper presents an overview of our findings on the effects of individualisation on the academic performance of college students. 
METHOD

We used standard procedures in our meta-analysis. To locate studies and reviews of individualised instruction, we searched several library data bases by computer. We then used the bibliographies in the research studies and in the reviews to locate additional articles on individualised instruction. Finally, we described features and outcome of each study in quantitative or quasi-quantitative terms.

For our meta-analysis of individualised instruction and college student achievement, we located 213 studies described in 209 reports. Each of the studies described a quantitative comparison of outcomes of individualised and conventional teaching in an actual college classroom. We first described the main features of the studies: the experimental design (type of subject assignment, presence of control for instructor and historical effects, control for scoring or author bias in achievement measures, etc); the course settings (course level, field of the course and level of the institution); and publication characteristics (form and year of publication). We also described the outcome of each study. As our index of the effect of individualised teaching on student achievement, we used Cohen's 'unit-free' measure of effect size $d$ (the number of standard-deviation-units separating performance of experimental and control groups).

Our methodology obviously owes a great deal to Glass's pioneering applications of meta-analysis (Smith and Glass, 1977; Glass and Smith, 1979). We see our methodology as different from Glass's, however, in two major ways. First, Glass typically uses far more effect sizes in his analyses than he has papers; he often uses several sets of findings from a single paper. To ensure greater independence among studies in our meta-analyses, we never use more than two results from a single paper. Our approach is similar to that of Gilbert, McPeek and Mosteller (1977). Second, Glass and his colleagues tend to include in a single analys is results from a wide array of outcome measures. In their meta-analysis of psychotherapy outcomes, for example, Smith and Glass (1977) grouped together outcome measures of different types; physiological measures, scores on projective devices, scores on self-report inventories, grade-point averages, and therapist ratings. Each of our analyses, on the other hand, has been restricted to a single variable that can be measured on a common unit.

RESULTS

Individualised instruction has a positive effect on student achievement in the studies we located. In 155 of the 213 studies included in this meta-analysis, examination performance in the individualised class was superior to examination performance in the conventional class; 58 studies favoured conventional instruction. of the 101 studies reporting a significant difference due to teaching method, 85 were in favour of individualisation, and 16 in favour of conventional teaching. If no overall generalisation about the effect of individualised teaching were possiblc, one would expect about half the cases to favour individualised instruction and half to favour conventional teaching. Instead, a clear majority of studies favoured individualised teaching. We were able, therefore, to reject the null hypothesis of no effect of individualisation on student achievement.

Continuous measures of effect size permit a more exact description of the influence of individualised teaching on examination performance. The average cohen's $d$ in the 213 studies was .33, with a standard deviation of .50 . Individualised instruction therefore raised examination performance of the typical student from the 50 th percentile to the 63rd percentile. Cohen (1977) calls effects of this magnitude 
small, and we agree with him. A further analysis showed that in the typical study the average score in the individualised class was 69.5 per cent on a 100-point examination, and was 65.4 per cent in the conventional class.

We carried out a further analysis of achievement findings to find out whether study features were related to study outcomes. Regression analys is showed that three factors influenced the outcomes of comparisons of individualised and conventional teaching (see Fig. 2.1). First, studies of individualised instruction that involved PSI produced clearer results than other studies. The typical size effect in the PSI studies was .56; the typical effect size in other studies was .24. Second, the beneficial effects of individualised instruction were clearer in comparisons which did not control for instructor effects. The difference between individualised and conventional instruction averaged .27 standard deviations in studies where both individualised and conventional classes were presented by the same teacher; the difference averaged .45 standard-deviation-units in other studies. This is counterintuitive. Looser experimental control would be expected to produce greater absoZute differences between experimental and control groups; looser control would not be expected to produce stronger support for the effectiveness of one of the methods. Finally, differences between experimental and control groups were less in thuse disciplines usually thought of as the hard sciences.

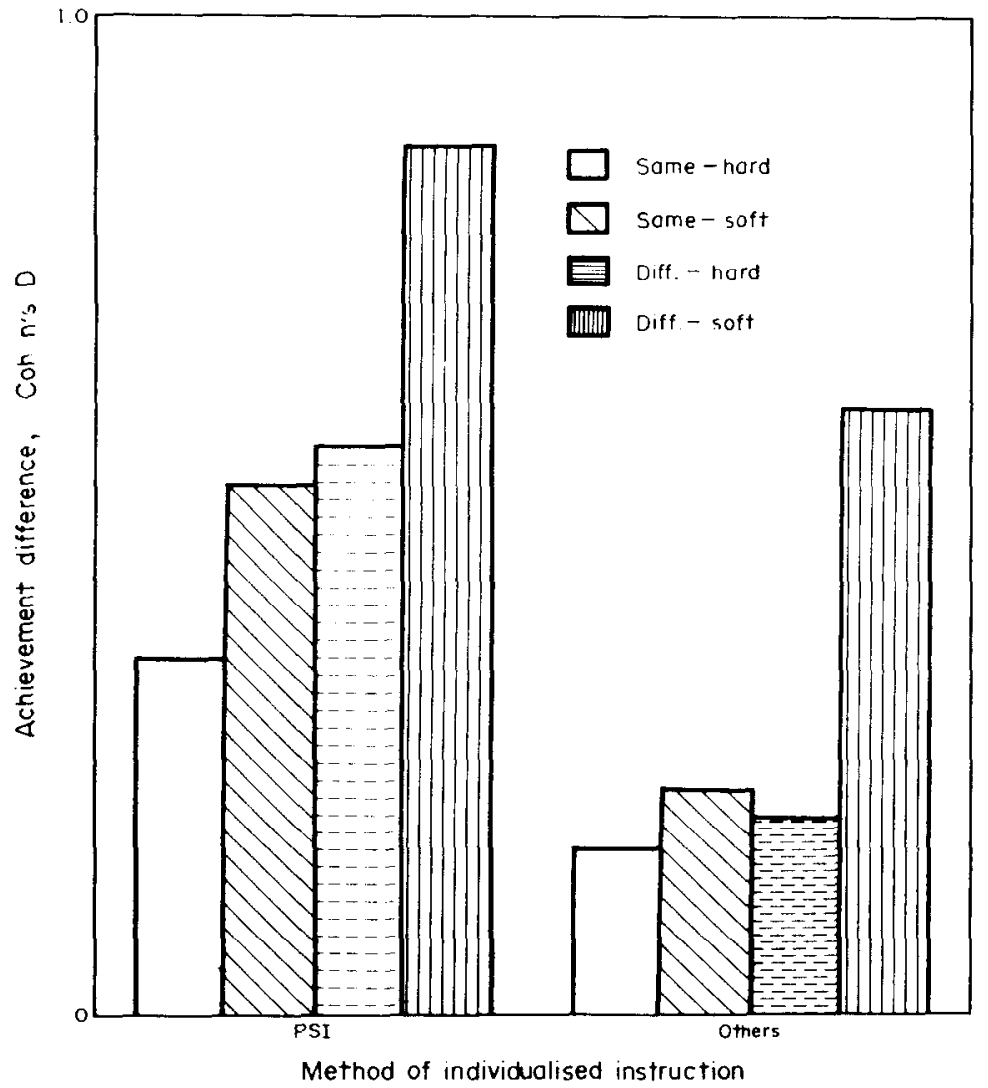

Fig. 2.1 Comparison of individualised and conventional teaching 


\section{SUMMARY}

A meta-analysis of 213 comparisons of individualised and conventional college teaching showed that individualisation generally produces small but significant improvements in student achievement. Effects were larger in studies where:

a. individualisation was achieved though Keller's Personalised System of Instmuction.

b. different instructors were responsible for the individualised and control sections of a course; and

c. individualised courses were not in the 'hard' sciences. 